Manuscript to Curr. Opin. Genet. Dev.

\title{
Role of autophagy in embryogenesis
}

Yoh Wada ${ }^{1}$, Ge-Hong Sun-Wada ${ }^{2}$, Nobuyuki Kawamura ${ }^{2}$, and Minako Aoyama ${ }^{1}$

1. Division of Biological Sciences, Institute of Scientific and Industrial Research, Osaka University, Mihogaoka 8-1, Ibaraki, Osaka 567-0047, Japan

2. Faculty of Pharmaceutical Sciences, Doshisha Women's College of Liberal Arts, Kohdo, Kyotanabe, Kyoto 610-0395, Japan

Corresponding author: Wada, Yoh (yohwada@sanken.osaka-u.ac.jp) 


\section{Abstract}

Eukaryotes have evolved multiple mechanisms for inactivating macromolecules in order to maintain their functionality. Autophagy-the process of self-eating-leads to the degradation of cytoplasmic components for the dynamic remodeling of subcellular compartments, turnover and recycling of macromolecules, and regulation of cellular activity through the control of specific intracellular signaling pathways. This fundamental process is also implicated in systemic response to starvation and immune challenges, as well as anti-tumorigenesis and anti-senescence. Recent studies have also highlighted an important role for autophagy in embryonic development. In this review, we discuss the emerging evidence for the varied functions of autophagy at different stages of development, with an emphasis on the early events of embryogenesis.

\section{Introduction}

During embryogenesis, the biosynthesis and degradation of molecules is essential for reorganizing cellular components and altering activities in response to dynamic developmental programs. Even in unicellular organisms-where the dilution of cellular content through cell division allows molecular turnover to be accomplished by transcriptional switching - a dedicated mechanism for inactivating existing proteins is employed: one of the best examples of this is in cell cycle progression. In multicellular organisms, the acquisition of tissue- and cell-specific functions through differentiation often occurs without cell division; thus, degradation of molecules is a prerequisite for development and maintenance of cellular architecture.

Inactivation and/or degradation of molecules is achieved through multiple mechanisms including ubiquitin-mediated proteasomal degradation[1], 
compartmentalization into multivesicular bodies [2], and lysosomal degradation[3] . In addition to these intracellular mechanisms, extracellular proteases, ligand antagonists, and sequestration by the extracellular components can also contribute to these functions [4-6]. Autophagic degradation is a cellular process in which organelles and components of cytosol are targeted to lysosomal compartments, where they are decomposed into small molecules that are then recycled. This recycling mechanism allows cells to conserve their limited resources by minimizing the costs associated with biosynthesis or acquiring resources from the environment. In addition to nutritional benefits, autophagy is thought to have an active role in regulating various cellular and tissue functions. Proteins are generally stable under ambient conditions; however, in any biological system, protein turnover is critical for rapid response to environmental changes as well as internal demands such as cell cycle progression. As an inherently dynamic process, embryonic development is highly dependent upon the regulation of intracellular and extracellular activities by proteins. Autophagy is implicated in many pathophysiological processes in multicellular organisms, including neural degeneration, cancer metastasis, and tissue remodeling, which are discussed in other comprehensive reviews $[7,8]$; the present discussion focuses on the role of autophagy during embryogenesis.

\section{Modes of autophagy}

Autophagy occurs through various mechanisms, i.e., micro-, macro-, and chaperone-mediated autophagy (Fig. 1). This classification is based on the manner in which substrates are delivered to the lysosomal compartment. Microautophagy involves unique membrane dynamics $[9,10]$; the lysosome, which often exhibits a giant vacuolar morphology, engulfs a portion of the cytosol as well as organelles through membrane invagination, delivering the 
substrates inwardly into the lumen of vacuolar lysosomes where various hydrolyses are enriched. This 'eating of cytosol/organelles by lysosome' release a single membrane encircled spherical body inside the lysosome, where the substrates destined to be degraded.

Macroautophagy progresses in a different fashion [11]. Initially, a cup-shaped membranous organelle emerges and encircles a portion of the cytosol, which often includes various organelles. This results in the formation of spherical bodies termed autophagosomes, in which a double membrane sequesters the compartmentalized material. Autophagosomes are directed to lysosomes, where their outer membrane fuses with the limiting membrane of lysosomes to release a single-membrane bound structures (autophagic bodies) into the lumen [12]. The acidic environment inside the lysosome promotes the digestion of macromolecules by hydrolyses, releasing amino acids, fatty acids, carbohydrates, and inorganic ions $[13,14]$. The molecular mechanism governing macroautophagy is highly conserved among eukaryotes; autophagy-related Atg proteins genetically identified in yeast [15] play a central role in the membrane dynamics.

A third type of autophagy is chaperone-mediated, and is distinguished by the fact that it does not involve membrane-membrane interactions, but instead translocates substrates across the lysosomal membrane from the cytosolic to luminal side using specific machinery.

\section{Microautophagy in embryogenesis}

The mechanism and physiology of microautophagy has been well studied in yeasts. Peroxisomes, which are carbohydrate-metabolizing organelles, are delivered to the lysosome/vacuoles via a microautophagic mechanism [16]. Peroxisome digestion is accompanied by rapid changes in cell physiology: cells grown with methanol as a carbon and energy source are enriched in peroxisomes, where 
methanol-metabolizing enzymes are concentrated; these disappear when more nutritious carbon sources such as glucose become available. Peroxisomes are then engulfed by vacuoles by pexophagy, a form of microautophagy. This conversion enables methanol-assimilating yeasts to survive in carbon-poor environments in open fields [17].

We recently showed that endosomes are internalized into large vacuoles by microautophagy around the time of gastrulation in mouse embryos. At this developmental stage, the embryo has a cylindrical structure in which the epiblast and extraembryonic ectoderm are surrounded by the visceral endoderm (VE), a stratified epithelium that separates the embryo proper from maternal tissues and serves as a tight barrier through which nutrients must be transported. The VE is therefore highly active in endocytosis and has a well-developed apparatus for this purpose (Figure 2). VE cells have large vacuoles on the apical side of the nucleus facing the maternal tissue. These apical vacuoles express lysosomal marker proteins including lysosomal-associated membrane protein (lamp) 1 and 2 and cathepsin proteinases, and accumulate endocytic tracers such as immunoglobulins and dextrans, suggesting that in undifferentiated cells, they are the functional equivalent of lysosomes.

However, the membrane dynamics of apical vacuoles are distinct from those of canonical lysosomes. Observations from live embryo imaging and pulse-chase experiments examining the endocytic process have revealed that 1) endosome-like structures as well as Rab7--a key cytosolic regulator of membrane trafficking at late stages of the endocytic pathway--are observed inside apical vacuoles; 2) endosomes and the apical vacuoles in which they resided ultimately merged their contents, a process that was blocked by a pharmacological inhibitor of lysosomal acid lipase; and 3) endosomes within apical vacuoles are bound by double membranes. Taken together, these lines of evidence suggest that 
endosome delivery occurs via microautophagy (Figure 3); unlike macroautophagy or canonical vesicular transport, this endosome-lysosome interaction involves neither formation of autophagosomes by engulfing membranes nor fusion between endosomal and lysosomal membranes [18][19].

The assembly of large apical vacuoles in VE depends upon the small GTP-binding protein Rab7 and mVam2 subunit of the membrane fusion machinery [18][20]. These proteins are structurally and functionally well conserved. Genetic deletion of Rab7 and mVam2 leads to fragmentation of vacuoles in yeast cells, as well as in the mouse VE [21]. In mVam2-deficient cells in mice, endocytic markers are concentrated in organelles that have features of the late endosome but do not reach the lamp2-positive lysosomal compartment. This suggests that the final stage of endocytosis is blocked, while earlier stages of endocytosis, i.e., initial internalization from the cell surface and compartmentalization in early endosome-like structures are less affected by the loss of $m V a m 2$ function.

Subcellular defects are associated with developmental phenotypes in the mVam2-deficient gastrulae, which develop the extraembryonic mesodermal tissues but fail to organize the embryonic mesoderm and neural ectoderm, producing a severe anterior-truncation phenotype. Prior to gastrulation, mVam2-deficient embryos exhibit ectopic activation of the bone morphogenetic protein (BMP)/Smad signaling: embryos accumulate BMP receptors at the cell surface as well as in the apical cytoplasm, suggesting that activated receptors continue to transmit signal to intracellular mediators; indeed, mouse embryonic fibroblasts (MEFs) derived from mutants are defective in the termination of BMP signaling. These results imply that the translocation of activated receptors to the late endosome and lysosome, and their timely termination--dependent on microautophagic delivery--plays a critical role in the spatial restriction of BMP signaling during tissue patterning [20] [22]. 
As stated, the VE has large apical vacuoles, which are atypical subcellular structures in animal cells that are characterized by more fragmented lysosomes [21]. Ileal epithelial cells in newborn rodents often develop large vacuoles resembling those in the VE [23]; however, it is unclear whether these are associated with microautophagy in the ileum.

Under certain circumstances, enlarged lysosomes have been observed in animal cells. It has been shown that the swelling of endosomal compartments can be induced by pharmacological inhibition of phosphatidyl inositol metabolism [24]. Genetic ablation of phosphatidylinositol (PI) phosphate kinase III (PIPKIII), the sole enzyme responsible for converting $\mathrm{PI}$ 3-phosphate (PI3P) into $\mathrm{PI}$

$(3,5)$-bisphosphate $\left[\mathrm{PI}(3,5) \mathrm{P}_{2}\right]$ in the endosomal membrane, results in the formation of giant vacuoles in MEFs [25]. The metabolism of PI3P to $\mathrm{PI}(3,5) \mathrm{P}_{2}$ is essential for vesicle budding from endosomes as well as invagination of the endosomal limiting membrane into the lumen during the formation of multivesicular late endosomes [26]; thus, its disruption has no effect on incoming membranes but causes a severe reduction in outflow and homeostasis, resulting in the accumulation of limiting membranes of endosomal compartments. These observations suggest that a balance between membrane influx and outflow is an important determinant of organelle size, although the mechanism underlying this process remains to be elucidated.

PI3P conversion is also required for microautophagy in the VE [25]. In PIPKIII mutant embryos, gigantic vacuoles with few internal endosomes/vesicles develop in the VE. It is worth noting that these differ from the apical vacuoles of wild-type cells, and exhibit characteristics of earlier compartments in the endocytic pathway. PIPKIII-deficient embryos have severe defects in gastrulation, failing to organize mesodermal structures and remaining as egg cylinders at embryonic day 7.5 , regenerating one day later. This provides further evidence that the correct 
assembly of vacuoles and microautophagy are essential for early embryonic development.

\section{Macroautophagy during embryogenesis}

Perinatal lethality is observed in mice deficient for Atg5 or Atg7—which are essential for the elongation of immature autophagosome membranes-due to multiple organ malfunction $[27,28]$. After birth, the delivery of nutrition through the maternal circulation is replaced by suckling, and during this transition, nutritional deficiencies are overcome by autophagy. Genetic studies have also revealed the importance of macroautophagy during early stages of embryogenesis, when maternally delivered Atg5 mRNA/protein provides sufficient function for development within a few days of fertilization; indeed, zygotic deletion of Atg5 leads to defective embryonic growth, where cell division is initiated but blastocysts are unable to form; these results imply that autophagy is required for this process, possibly to provide nutrition [29]. Recently, cargo-specific autophagy has also been reported to assist in quality control of essential cellular constituents [30,31]. An open question is whether the degradation of specific proteins/mRNAs/organelles is essential for development, or if bulk degradation of maternally inherited molecules contributes to the rebuilding of zygotic components.

In eukaryotes, maternal mitochondrial genetic information is inherited and the concomitant, selective inactivation of the paternal mitochondrial genome is a major event in early developmental stages; however, the underlying mechanisms are poorly understood. Recently, macroautophagy was shown to be involved in this process in nematodes; genetic ablation or RNA interference of autophagy-related genes produced defects in the degradation of paternal mitochondria [32]. Evidence from mice on the role of autophagic elimination of mitochondrial genetic information has been contradictory [33] [34], suggesting that 
species-specific mechanisms exist for mitochondrial and chloroplast DNA elimination, which involve nucleases, ubiquitin-mediated degradation, DNA methylation, and other as yet unidentified processes [35].

\section{Macroautophagy in organogenesis}

Atg5 or 7 function is not a prerequisite for morphogenesis, except during blastocyst formation at the earliest stages of embryogenesis [27] [29]. In contrast, beclin--belonging to another class of macroautophagy-related protein--is required for developmental progression. Beclin-deficient embryos develop into egg cylinders but are arrested at gastrulation [36]. In addition, deletion of activating molecule in beclin-regulated autophagy (ambra) 1, a beclin-interacting protein, produces severe defects in neural tube closure in mice, as well as multiple defects in organogenesis in zebrafish [37] [38]. Beclin and ambra 1 form a large complex that regulates macroautophagy and the endocytic pathway [39] and is implicated in retrograde membrane flow from the endolysosome to the Golgi apparatus [40]. It remains unclear whether defects in macroautophagy or endocytic trafficking or both are responsible for the observed mutant phenotypes; however, gastrulation is compromised in mice lacking Vps52, a mediator of retrograde transport [41] [42].

The beclin complex regulates cell proliferation and apoptosis, and is therefore a hub for various cellular processes including autophagy, endocytosis, and cell fate determination; the neuronal phenotype arises from the fact that neural closure involves massive and dynamic cellular reorganization and apoptosis within a relatively short period $[43,44]$.

\section{Conclusion and perspectives}

In the last decade, it has become increasingly clear that micro- and macroautophagy play essential roles during early embryogenesis and 
organogenesis, as well as in the homeostatic maintenance of adult tissues. Future studies of mutants defective in endocytosis and related processes will provide greater insight into mechanisms of autophagic regulation of cellular and tissue architecture in multicellular organisms.

\section{Acknowledgements}

The authors thank colleagues in the fields of development and cell biology for fruitful discussions and exchanges of ideas. The work performed by the authors was supported by the Core Research for Evolutional Science and Technology, Japan Science and Technology Agency, and the Ministry of Education, Culture, Sports, Science and Technology (Japan).

\section{References}

1. Welchman RL, Gordon C, Mayer RJ: Ubiquitin and ubiquitin-like proteins as multifunctional signals. Nat Rev Mol Cell Biol 2005, 6:599-609.

2. Seto ES, Bellen HJ, Lloyd TE: When cell biology meets development: endocytic regulation of signaling pathways. Genes Dev. 2002, 16:1314-1336.

3. Luzio JP, Pryor PR, Bright NA: Lysosomes: fusion and function. Nat. Rev. Mol. Cell Biol. 2007, 8:622-632.

4. Branford WW, Yost HJ: Nodal signaling: CrypticLefty mechanism of antagonism decoded. Curr Biol. 2004, 14:R341-343.

5. Ben-Haim N, Lu C, Guzman-Ayala M, Pescatore L, Mesnard D, Bischofberger M, Naef F, Robertson EJ, Constam DB: The Nodal precursor acting via activin receptors induces mesoderm by maintaining a source of its convertases and BMP4. Dev. Cell 2006, 11:313-323. 
6. Shimokawa K, Kimura-Yoshida C, Nagai N, Mukai K, Matsubara K, Watanabe H, Matsuda Y, Mochida K, Matsuo I: Cell surface heparan sulfate chains regulate local reception of FGF signaling in the mouse embryo. Dev Cell 2011, 21:257-272.

7. Uchiyama Y, Shibata M, Koike M, Yoshimura K, Sasaki M:

Autophagy-physiology and pathophysiology. Histochem Cell Biol 2008, 129:407-420.

8. Mizushima N, Levine B: Autophagy in mammalian development and differentiation. Nat Cell Biol 2010, 12:823-830.

9. Marzella L, Ahlberg J, Glaumann H: Autophagy, heterophagy, microautophagy and crinophagy as the means for intracellular degradation. Virchows Arch B Cell Pathol Incl Mol Pathol 1981, 36:219-234.

10. Sahu R, Kaushik S, Clement CC, Cannizzo ES, Scharf B, Follenzi A, Potolicchio I, Nieves E, Cuervo AM, Santambrogio L: Microautophagy of cytosolic proteins by late endosomes. Dev Cell 2011, 20:131-139.

11. Baba M, Takeshige K, Baba N, Ohsumi Y: Ultrastructural analysis of the autophagic process in yeast: detection of autophagosomes and their characterization. J. Cell Biol. 1994, 124:903-913.

12. Takeshige K, Baba M, Tsuboi S, Noda T, Ohsumi Y: Autophagy in yeast demonstrated with proteinase-deficient mutants and conditions for its induction. J. Cell Biol. 1992, 119:301-311.

13. Nakamura N, Matsuura A, Wada Y, Ohsumi Y: Acidification of vacuoles is required for autophagic degradation in the yeast, Saccharomyces cerevisiae. J Biochem 1997, 121:338-344.

14. Kinouchi K, Ichihara A, Sano M, Sun-Wada GH, Wada Y, Kurauchi-Mito A, Bokuda K, Narita T, Oshima Y, Sakoda M, et al.: The (pro)renin 
receptor/ATP6AP2 is essential for vacuolar $\mathrm{H}^{+-} \mathrm{ATPase}$ assembly in murine cardiomyocytes. Circ Res 2010, 107:30-34.

15. Tsukada M, Ohsumi Y: Isolation and characterization of autophagy-defective mutants of Saccharomyces cerevisiae FEBS Lett 1993, 333:169-174.

16. Sakai Y, Koller A, Rangell LK, Keller GA, Subramani S: Peroxisome degradation by microautophagy in Pichia pastoris: identification of specific steps and morphological intermediates. J Cell Biol 1998, 141:625-636.

17. Kawaguchi K, Yurimoto H, Oku M, Sakai Y: Yeast methylotrophy and autophagy in a methanol-oscillating environment on growing Arabidopsis thaliana leaves. PLoS One 2011, 6:e25257.

18. Kawamura N, Sun-Wada G-H, Aoyama M, Harada A, Takasuga S, Sasaki T, Wada Y: Delivery of endosomes to lysosomes via microautophagy in the visceral endoderm of mouse embryos. Nat Commun 2012, 3:1071.

19. Wada Y, Sun-Wada GH, Kawamura N: Microautophagy in the visceral endoderm is essential for mouse early development. Autophagy 2013, 9:252-254.

20. Aoyama M, Sun-Wada G-H, Yamamoto A, Yamamoto M, Hamada H, Wada Y: Spatial restriction of bone morphogenetic protein signaling in mouse gastrula through the mVam2-dependent endocytic pathway. Dev Cell 2012, 22:1163-1175.

21. Wada Y: Vacuoles in mammals: A subcellular structure indispensable for early embryogenesis. BioArchitecture 2013, 3:13-19.

22. Wada Y, Sun-Wada G-H: Positive and negative regulation of developmental signaling by the endocytic pathway. Curr Opin Genet Dev 2013, 23:391-398. 
23. Wissig SL, Graney DO: Membrane modifications in the apical endocytic complex of ileal epithelial cells. J. Cell Biol. 1968, 39:564-579.

24. Jefferies HB, Cooke FT, Jat P, Boucheron C, Koizumi T, Hayakawa M, Kaizawa H, Ohishi T, Workman P, Waterfield MD, et al.: A selective PIKfyve inhibitor blocks PtdIns $(3,5) \mathrm{P}(2)$ production and disrupts endomembrane transport and retroviral budding. EMBO Rep 2008, 9:164-170.

25. Takasuga S, Horie Y, Sasaki J, Sun-Wada GH, Kawamura N, Iizuka R, Mizuno K, Eguchi S, Kofuji S, Kimura H, et al.: Critical roles of type III phosphatidylinositol phosphate kinase in murine embryonic visceral endoderm and adult intestine. Proc Natl Acad Sci U SA 2013, 110:1726-1731.

26. Sasaki T, Takasuga S, Sasaki J, Kofuji S, Eguchi S, Yamazaki M, Suzuki A: Mammalian phosphoinositide kinases and phosphatases. Prog Lipid Res 2009, 48:307-343.

27. Kuma A, Hatano M, Matsui M, Yamamoto A, Nakaya H, Yoshimori T, Ohsumi Y, Tokuhisa T, Mizushima N: The role of autophagy during the early neonatal starvation period. Nature 2004, 432:1032-1036.

28. Komatsu M, Waguri S, Ueno T, Iwata J, Murata S, Tanida I, Ezaki J, Mizushima N, Ohsumi Y, Uchiyama Y, et al.: Impairment of starvation-induced and constitutive autophagy in Atg7-deficient mice. $J$ Cell Biol 2005, 169:425-434.

29. Tsukamoto S, Kuma A, Murakami M, Kishi C, Yamamoto A, Mizushima N: Autophagy is essential for preimplantation development of mouse embryos. Science 2008, 321:117-120.

30. Riley BE, Kaiser SE, Shaler TA, Ng AC, Hara T, Hipp MS, Lage K, Xavier RJ, Ryu KY, Taguchi K, et al.: Ubiquitin accumulation in 
autophagy-deficient mice is dependent on the Nrf2-mediated stress response pathway: a potential role for protein aggregation in autophagic substrate selection. J Cell Biol 2010, 191:537-552.

31. Ichimura Y, Waguri S, Sou YS, Kageyama S, Hasegawa J, Ishimura R, Saito T, Yang Y, Kouno T, Fukutomi T, et al.: Phosphorylation of $\mathrm{p} 62$ activates the Keap1-Nrf2 pathway during selective autophagy. Mol Cell 2013, 51:618-631.

32. Sato M, Sato K: Degradation of paternal mitochondria by fertilization-triggered autophagy in C. elegans embryos. Science 2011, 334:1141-1144.

33. Al Rawi S, Louvet-Vallee S, Djeddi A, Sachse M, Culetto E, Hajjar C, Boyd L, Legouis R, Galy V: Postfertilization autophagy of sperm organelles prevents paternal mitochondrial DNA transmission. Science 2011, 334:1144-1147.

34. Luo SM, Ge ZJ, Wang ZW, Jiang ZZ, Wang ZB, Ouyang YC, Hou Y, Schatten $\mathrm{H}$, Sun QY: Unique insights into maternal mitochondrial inheritance in mice. Proc Natl Acad Sci U SA 2013, 110:13038-13043.

35. Sato M, Sato K: Maternal inheritance of mitochondrial DNA by diverse mechanisms to eliminate paternal mitochondrial DNA. Biochim Biophys Acta 2013, 1833:1979-1984.

36. Yue Z, Jin S, Yang C, Levine AJ, Heintz N: Beclin 1, an autophagy gene essential for early embryonic development, is a haploinsufficient tumor suppressor. Proc. Natl. Acad. Sci. U S A 2003, 100:15077-15082.

37. Maria Fimia G, Stoykova A, Romagnoli A, Giunta L, Di Bartolomeo S, Nardacci R, Corazzari M, Fuoco C, Ucar A, Schwartz P, et al.: Ambra1 regulates autophagy and development of the nervous system. Nature 2007, 447:1121-1125. 
38. Benato F, Skobo T, Gioacchini G, Moro I, Ciccosanti F, Piacentini M, Fimia GM, Carnevali O, Dalla Valle L: Ambra1 knockdown in zebrafish leads to incomplete development due to severe defects in organogenesis. Autophagy 2013, 9:476-495.

39. Liang C, Lee JS, Inn KS, Gack MU, Li Q, Roberts EA, Vergne I, Deretic V, Feng P, Akazawa C, et al.: Beclin1-binding UVRAG targets the class $\mathrm{C}$ Vps complex to coordinate autophagosome maturation and endocytic trafficking. Nat Cell Biol 2008, 10:776-787.

40. Itakura E, Kishi C, Inoue K, Mizushima N: Beclin 1 forms two distinct phosphatidylinositol 3-kinase complexes with mammalian Atg14 and UVRAG. Mol Biol Cell 2008, 19:5360-5372.

41. Sugimoto M, Kondo M, Hirose M, Suzuki M, Mekada K, Abe T, Kiyonari H, Ogura A, Takagi N, Artzt K, et al.: Molecular identification of $\mathrm{t}(\mathrm{w} 5)$ : Vps52 promotes pluripotential cell differentiation through cell-cell interactions. Cell Rep 2012, 2:1363-1374.

42. Conibear E, Stevens TH: Vps52p, Vps53p, and Vps54p form a novel multisubunit complex required for protein sorting at the yeast late Golgi. Mol. Biol.. Cell 2000, 11:305-323.

43. Yamaguchi Y, Shinotsuka N, Nonomura K, Takemoto K, Kuida K, Yosida H, Miura M: Live imaging of apoptosis in a novel transgenic mouse highlights its role in neural tube closure. J Cell Biol 2011, 195:1047-1060. 44. Nonomura K, Yamaguchi Y, Hamachi M, Koike M, Uchiyama Y, Nakazato K, Mochizuki A, Sakaue-Sawano A, Miyawaki A, Yoshida H, et al.: Local Apoptosis Modulates Early Mammalian Brain Development through the Elimination of Morphogen-Producing Cells. Dev Cell 2013, 27:621-634.

\section{Notes on the selected references}


$10^{*}$. Sahu et al *

This study showed that delivery of cytosolic proteins are delivered into the lumen of multivesicular endosomes occurs via the microautophagy.

$11^{* *}$. Takeshige et al., and $12^{* *}$ Baba et al., **

These two pioneering studies on autophagy showed that yeast cells accumulate autophagic bodies inside the vacuole during nutrient starvation, which are derived from autophagosome-a membrane structure encircling cytoplasm--, showing membrane dynamics of macroautophagy.

\section{$15^{\star}$ Tsukada and Ohsumi}

This study identified over a dozen genes encoding autophagy-related proteins (Atg) involved in macroautophagy, and applied molecular biological approaches to the dissection of underlying mechanisms. Subsequent studies showed that Atg genes are highly conserved among eukaryotes including in mammals, plants, and yeast. These findings enabled reverse genetic studies in mice and other organisms that revealed various pathologies associated with autophagy deficiency.

\section{$18^{\star \star}$ Kawamura et al}

This study demonstrated that microautophagy is involved in the assembly of large apical vacuoles using pulse-chase experiments to label endocytic markers, and electron and fluorescence microscopy in mouse embryos. Microautophagy was shown to require the small GTP-binding protein Rab7, which is a known regulator of late stages of endocytosis. Genetic ablation of Rab7 resulted in defective gastrulation, indicating that subcellular and systemic events are tightly linked. 
$20^{* *}$ Aoyama et al

The mouse Vam2 (mVam2) protein was shown to be involved in microautophagy and the assembly of vacuoles in the visceral endoderm; mVam2-deficient embryos lacked apical vacuoles and were unable to complete gastrulation. Ectopic activation of bone morphogenetic protein/Smad signaling by microautophagy resulted in defects in embryogenesis.

$25^{\star *}$ Takasuga et al

This study showed that phosphatidyl inositol $(\mathrm{PI})$ metabolism is required for the proper assembly of apical vacuoles. A defect in the conversion of PI 3-phosphate to $\mathrm{PI}(3,5)$-bisphosphate resulted in the swelling of apical vacuoles, accompanied by developmental arrest at gastrulation. These findings highlighted the importance of the endocytic/autophagic pathway in normal gastrulation.

\section{$29^{* *}$ Tsukamoto et al}

After fertilization, maternally inherited molecules are replaced by those that are newly synthesized. This study showed that this switch is accompanied by the activation of macroautophagy. Depletion of the autophagy-related protein Atg5 has no effect on fertilization, but loss of both maternal and zygotic Atg5 caused developmental arrest at the 4-8-cell stage, demonstrating that macroautophagy immediately after fertilization is essential for the progression to blastocyst stages.

\section{$32 *$ Sato and Sato}

Uniparental inheritance of mitochondrial or plastid genetic information is common to eukaryotes. In C. elegans, mitochondria from sperm cytoplasm are rapidly degraded upon fertilization, which was shown in this study to be inhibited in the absence of macroautophagy, leading to developmental arrest. These results 
showed that substrate-specific macroautophagy prevents the transmission of the paternal mitochondrial genome in C. elegans. 


\section{Figure legends}

Figure 1. Modes of micro-, macro-, and chaperone-mediated autophagy. Autophagy, or self-eating, occurs through at least three different mechanisms. In microautophagy, lysosomal compartments invaginate and engulf a portion of the cytosol along with organelles, forming membrane-bound spherical bodies within lysosomes. Macroautophagy involves the formation of autophagosomes that subsequently fuse with the lysosome. In chaperone-mediated autophagy, substrates are translocated across the lysosome membrane and delivered directly into the lumen.

Figure 2. Morphology of the apical vacuoles (AV) in the visceral endoderm (VE) of the mouse embryo.

Wild-type VE cells have large vacuolar organelles in the cytoplasm on the apical side of the nucleus. Spherical endosomes with variable levels of staining with heavy metals can be seen between the vacuoles and the cell surface covered with microvilli. In contrast, VE cells in Rab7 ${ }^{-1}$ embryos lack apical vacuoles, accumulating instead numerous endosome-like vesicles throughout the cytoplasm.

\section{Figure 3. Membrane dynamics in the visceral endoderm of mouse embryos.}

In pulse-chase experiments, embryos were sequentially labeled with red and green fluorescent dextran at the gastrulation stage (embryonic day 6.5). The red dextran accumulated in apical vacuoles, while crescent-shaped early endosomes incorporated green-dextran and were visible immediately ( $5 \mathrm{~min}$ ) after beginning the chase, becoming more spherical in shape; endosomes were then surrounded by (15 $\mathrm{min}$ ) and merged with (30 $\mathrm{min})$ red dextran-containing vacuoles. This process occurred via microautophagy of endosomes by apical vacuoles. 
Figure 4. Developmental stages critically dependent on autophagy.

Micro- and macroautophagy are implicated in various stages of embryonic development. 


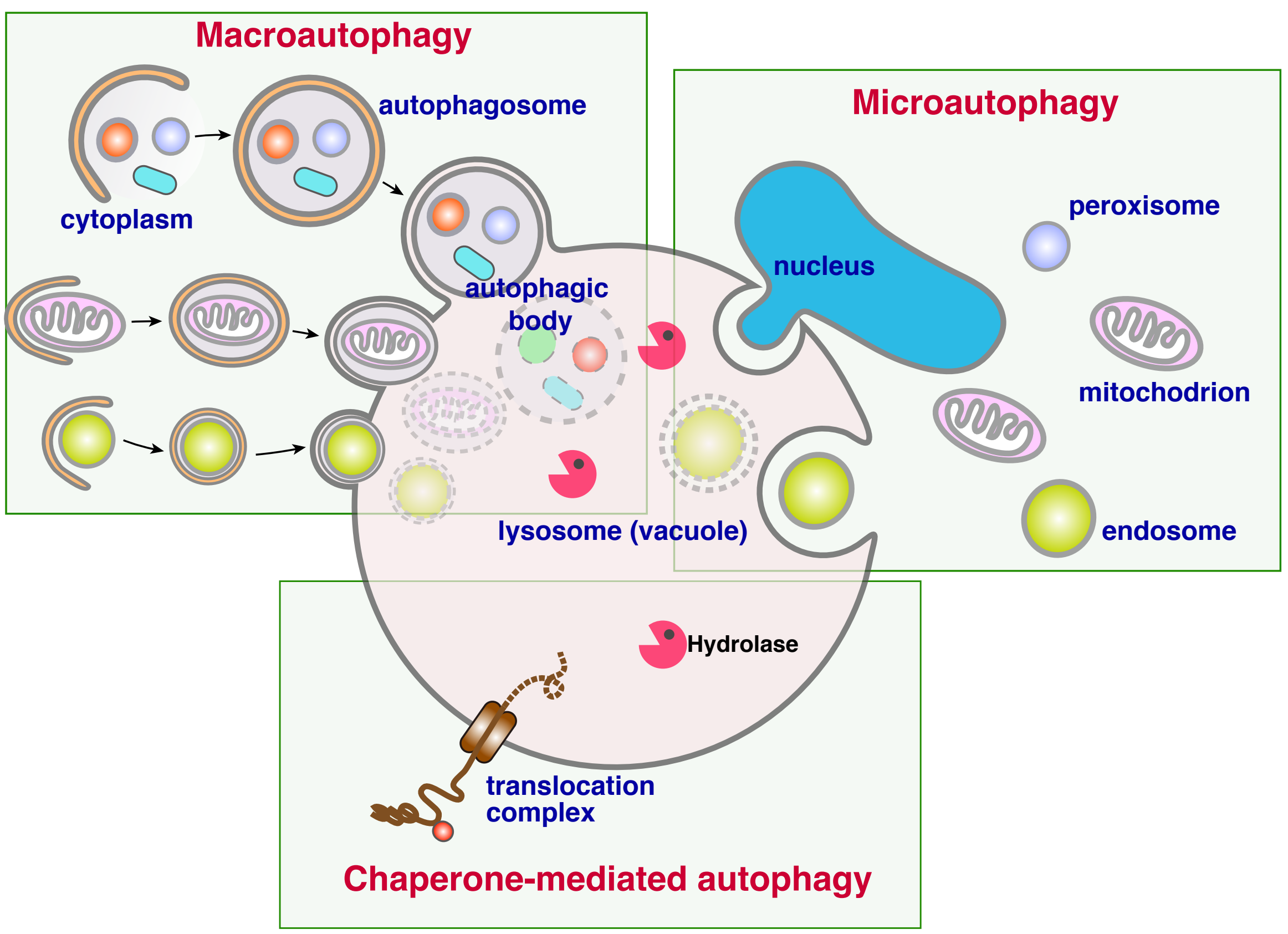




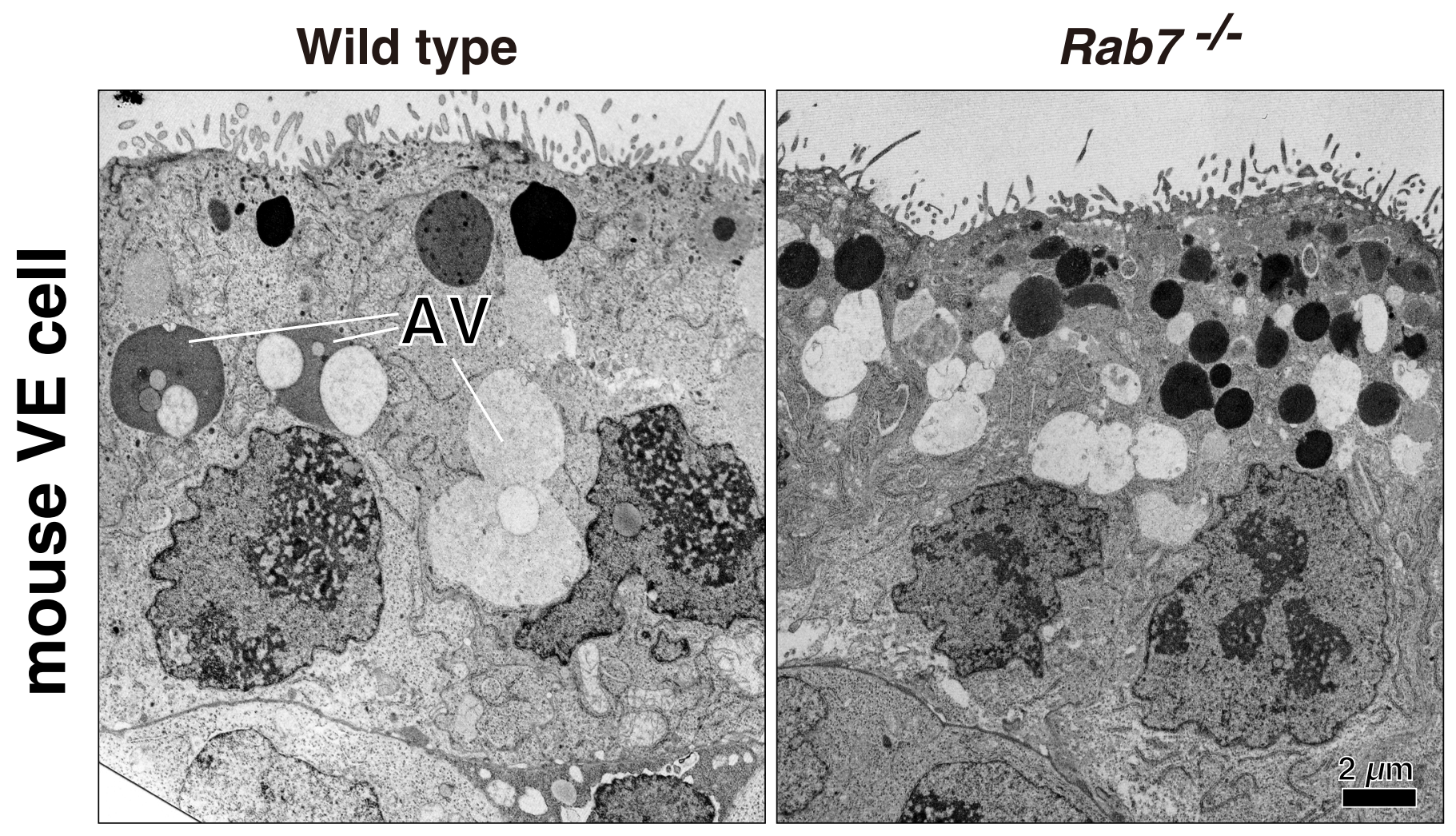




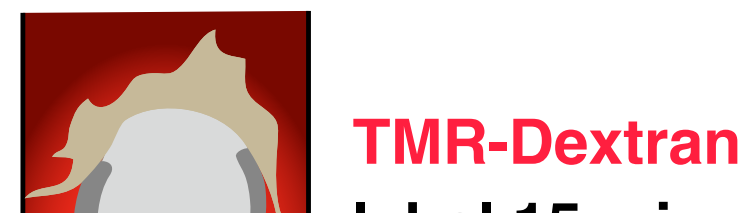
label 15 min chase 15 min

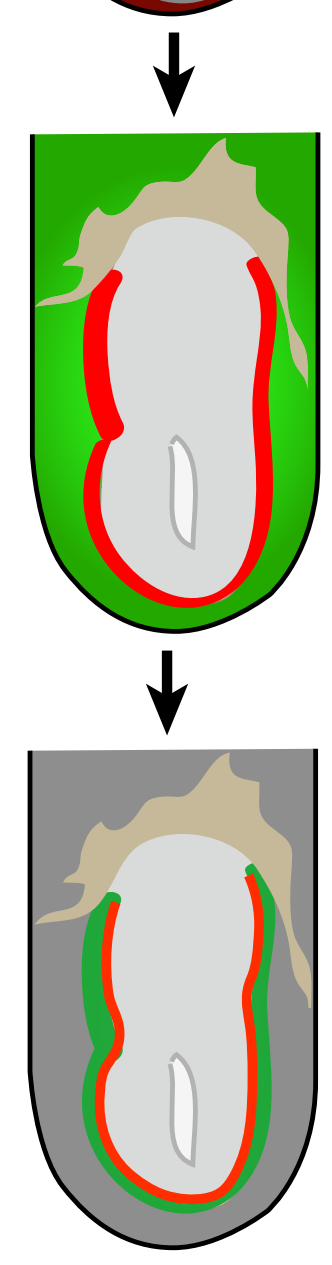

Chase

$5,15,30 \mathrm{~min}$

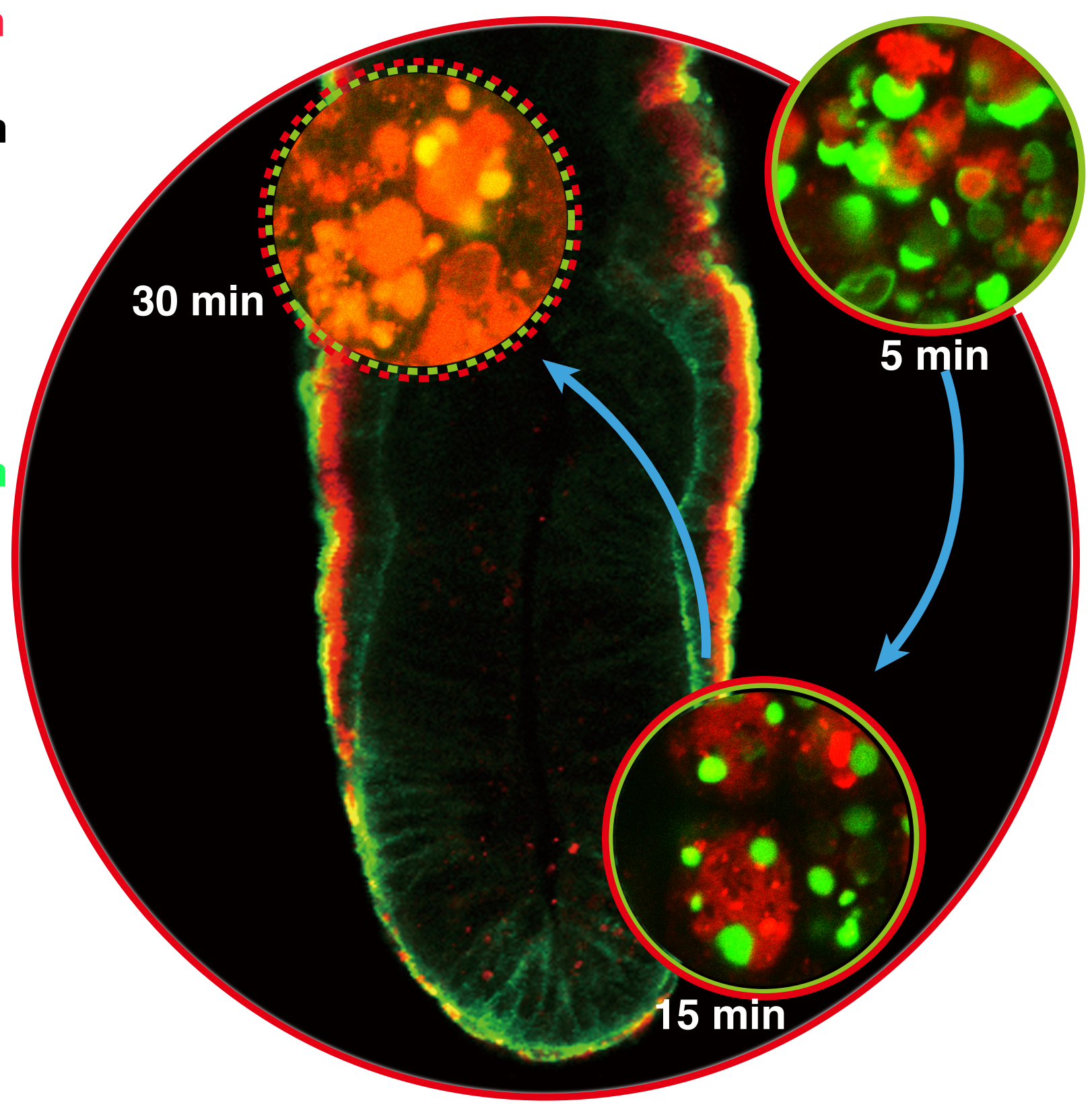




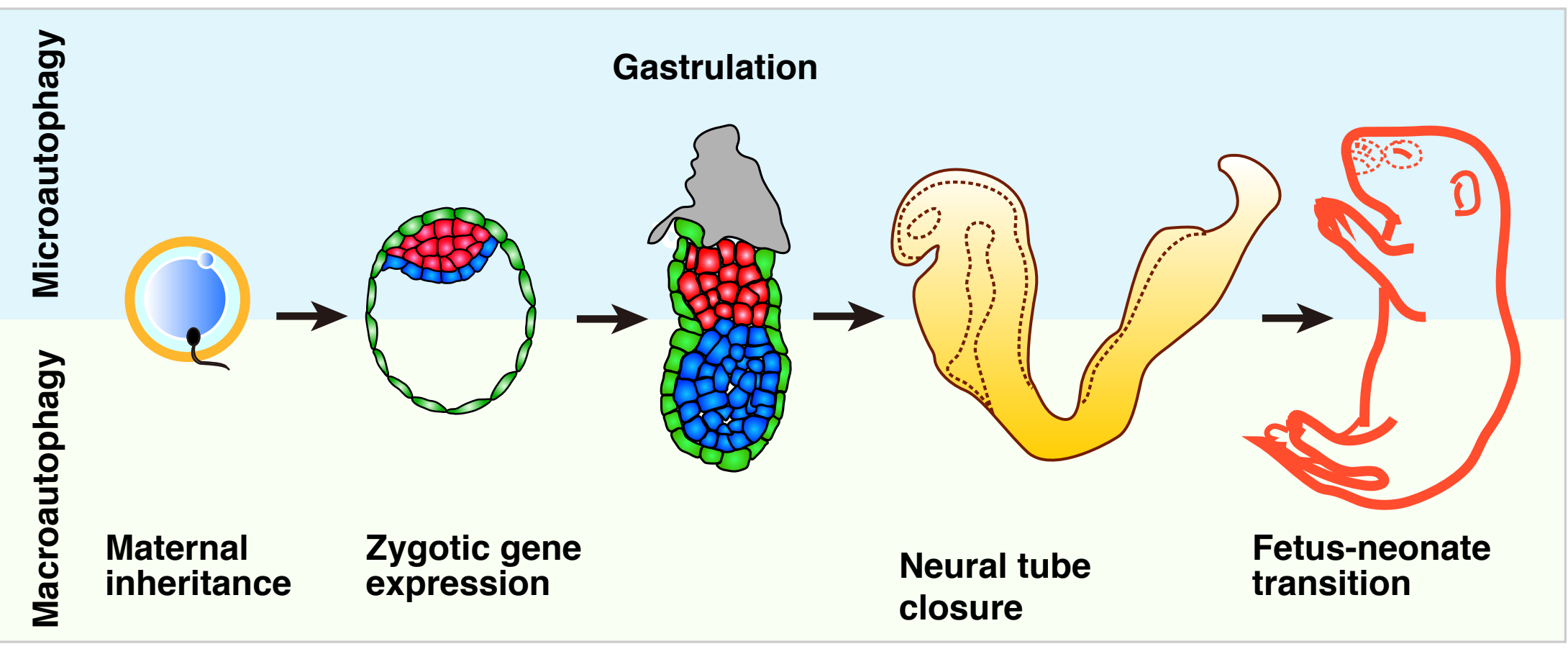

